\title{
Symmetric functions, codes of partitions and the KP hierarchy
}

\author{
S.R. Carrell • I.P. Goulden
}

Received: 27 February 2009 / Accepted: 17 November 2009 / Published online: 11 December 2009

(C) Springer Science+Business Media, LLC 2009

\begin{abstract}
We consider an operator of Bernstein for symmetric functions and give an explicit formula for its action on an arbitrary Schur function. This formula is given in a remarkably simple form when written in terms of some notation based on the code of a partition. As an application, we give a new and very simple proof of a classical result for the KP hierarchy, which involves the Plücker relations for Schur function coefficients in a $\tau$-function for the hierarchy. This proof is especially compact because we are able to restate the Plücker relations in a form that is symmetrical in terms of partition code notation.
\end{abstract}

Keywords Symmetric functions - Schur functions · Plücker relations · KP hierarchy $\cdot$ Combinatorial bijection $\cdot$ Partition code

\section{Introduction}

In Macdonald's fundamental book [10, p. 95] on symmetric functions, he considers the operators $B(t), B_{n}$ given by

$$
B(t):=\sum_{n \in \mathbb{Z}} B_{n} t^{n}:=\exp \left(\sum_{k \geq 1} \frac{t^{k}}{k} p_{k}\right) \exp \left(-\sum_{k \geq 1} t^{-k} \frac{\partial}{\partial p_{k}}\right),
$$

which he attributes to Bernstein [16, p. 69]. Here $t$ is an indeterminate, and $p_{k}$ is the $k$ th power sum symmetric function of a countable set of indeterminates (in which

S.R. Carrell · I.P. Goulden ( $\varangle)$

Department of Combinatorics and Optimization, University of Waterloo, Waterloo, Ontario, Canada e-mail: ipgoulden@uwaterloo.ca

S.R. Carrell

e-mail: srcarrell@uwaterloo.ca 
the power sums are symmetric). Of course, since the power sum symmetric functions $p_{1}, p_{2}, \ldots$ in a countable set of indeterminates are algebraically independent (see Sect. 3 for this and other basic results about symmetric functions), we can regard $p_{1}, p_{2}, \ldots$ as indeterminates. Hence we consider Bernstein's operators $B(t), B_{n}$ applied to formal power series in $p_{1}, p_{2}, \ldots$ Let the weight of the monomial $p_{1}^{i_{1}} p_{2}^{i_{2}} \ldots$ be given by $i_{1}+2 i_{2}+\cdots$. Then note that as a series in $t, B(t)$ goes to infinity in both directions, but that when it is applied to a monomial of weight $m$ in $p_{1}, p_{2}, \ldots$, the result is a Laurent series with minimum degree $-m$ in $t$.

The main result of this paper is an explicit expansion for the Laurent series that results from applying $B(t)$ to an arbitrary Schur symmetric function (where the Schur function is written as a polynomial in $p_{1}, p_{2}, \ldots$ with symmetric group characters as coefficients, as given in (8)). This result, appearing as Theorem 3.4, is given in a remarkably simple form by using an indexing notation for partitions that is natural in terms of the code of the partition.

For an application of our main result, we turn to the KP hierarchy of mathematical physics. By combining our main result and its dual form, we are able to give a new, simple proof (in Theorem 5.2) of the classical result for the KP hierarchy that relates the Schur symmetric function expansion of a $\tau$-function for the hierarchy and the Plücker relations for the coefficients in this expansion. This proof is made especially compact by our restatement (as Theorem 4.1) of the Plücker relations, in a highly symmetrical form in terms of the code notation.

The KP (Kadomtsev-Petviashvili) hierarchy is a completely integrable hierarchy that generalizes the KdV hierarchy (an integrable hierarchy is a family of partial differential equations which are simultaneously solved; for a comprehensive and concise account of the KP hierarchy with an emphasis on the view of physics, see Miwa, Jimbo, and Date [11]). Over the last two decades, there has been strong interest in the relationship between integrable hierarchies and moduli spaces of curves. This began with Witten's Conjecture [15] for the KdV equations, proved by Kontsevich [9] (and more recently by a number of others, including [8]). Pandharipande [13] conjectured that solutions to the Toda equations arose in a related context, which was proved by Okounkov [12], who also proved the more general result that a generating series for what he called double Hurwitz numbers satisfies the KP hierarchy. Kazarian and Lando [8], in their recent proof of Witten's conjecture, showed that it is implied by Okounkov's result for double Hurwitz numbers. More recently, Kazarian [7] has given a number of interesting results about the structure of solutions to the KP hierarchy. Combinatorial aspects of this connection have been studied by Goulden and Jackson [2].

The structure of this paper is as follows. In Sect. 2, we describe the notation for partitions (which index symmetric functions), their diagrams, and codes (in terms of the physics presentation in [11], the code is equivalent to a Maya diagram without the location parameter of charge). In Sect. 3, we describe the algebra of symmetric functions and give our main result, with a combinatorial proof in terms of the diagrams of partitions. Section 4 contains our symmetrical restatement of the Plücker relations. Finally, in Sect. 5, we give our new proof of the Schur-Plücker result for the KP hierarchy, together with a description of an equivalent system of partial differential equations for the KP hierarchy in terms of codes of partitions. 

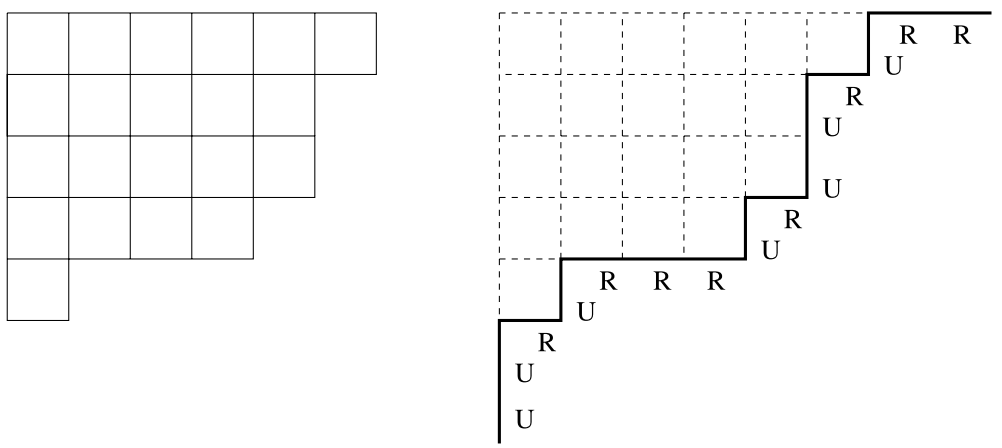

Fig. 1 The diagram and code for the partition $(6,5,5,4,1)$

\section{Partitions and codes}

We begin with some notation for partitions (see $[10,14])$. If $\lambda_{1}, \ldots, \lambda_{n}$ are integers with $\lambda_{1} \geq \cdots \geq \lambda_{n} \geq 1$ and $\lambda_{1}+\cdots+\lambda_{n}=d$, then $\lambda=\left(\lambda_{1}, \ldots, \lambda_{n}\right)$ is said to be a partition of $|\lambda|:=d$ (indicated by writing $\lambda \vdash d$ ) with $l(\lambda):=n$ parts. The empty list $\varepsilon$ of integers is to be regarded as a partition of $d=0$ with $n=0$ parts. Let $\mathcal{P}$ denote the set of all partitions. If $\lambda$ has $f_{j}$ parts equal to $j$ for $j=1, \ldots, d$, then, when convenient, we also write $\lambda=\left(d^{f_{d}}, \ldots, 1^{f_{1}}\right)$. Also, Aut $\lambda$ denotes the set of permutations of the $n$ positions that fix $\lambda$; therefore $\mid$ Aut $\lambda \mid=\prod_{j \geq 1} f_{j}$ !. The conjugate of $\lambda$ is the partition $\lambda^{\prime}=\left(\lambda_{1}^{\prime}, \ldots, \lambda_{m}^{\prime}\right)$, where $m=\lambda_{1}$, and $\lambda_{i}^{\prime}$ is equal to the number of parts $\lambda_{j}$ of $\lambda$ with $\lambda_{j} \geq i, i=1, \ldots, m$. The diagram of $\lambda$ is a left-justified array of juxtaposed unit squares, with $\lambda_{i}$ squares in the $i$ th row. For example, the diagram of the partition $(6,5,5,4,1)$ is given on the left-hand side of Fig. 1 . The diagram gives a compact geometrical description of the conjugate; the diagram of $\lambda^{\prime}$ is obtained by reflecting the diagram of $\lambda$ about the main diagonal.

The code of a partition is a two-way infinite binary string in two symbols, say U, R. The string is infinitely $\mathrm{U}$ to the left and infinitely $\mathrm{R}$ to the right, and represents a lattice path with unit up-steps (for " $U$ ") and unit right-steps (for "R"); the path starts moving up the $y$-axis towards the origin, ends moving out the $x$-axis away from the origin, and forms the lower right-hand boundary of the diagram of the partition, when the diagram is placed with its upper left-hand corner at the origin. An example is given on the right-hand side of Fig. 1, which gives us the string ...UURURRRURUURURR... as the code of the partition $(6,5,5,4,1)$ (see, e.g., [14, p. 467] for more on codes; in [14], the symbols in the code are 0,1 , but we prefer $\mathrm{U}, \mathrm{R}$ as more mnemonic.)

We let $\lambda^{(i)}, i \geq 1$, be the partition whose code is obtained from the code of the partition $\lambda$ by switching the $i$ th $\mathrm{R}$ (from the left) to $\mathrm{U}$. If $\lambda=\left(\lambda_{1}, \ldots, \lambda_{n}\right)$, then we immediately have

$$
\lambda^{(i)}=\left(\lambda_{1}-1, \ldots, \lambda_{j}-1, i-1, \lambda_{j+1}, \ldots \lambda_{n}\right),
$$

where $j$ is chosen (uniquely) from $0, \ldots, n$ so that $\lambda_{j} \geq i>\lambda_{j+1}$ (with the conventions that $\lambda_{n+1}=0$ and $\lambda_{0}=\infty$ ). Now define $u_{i}(\lambda)$ to be the number of up-steps $\mathrm{U}$ that follow the $i$ th right-step R from the left in the code of $\lambda$. Then note that $u_{i}(\lambda)=j$ 
Fig. 2 The skew diagram $(6,5,5,4,1)-(3,3,2,1)$

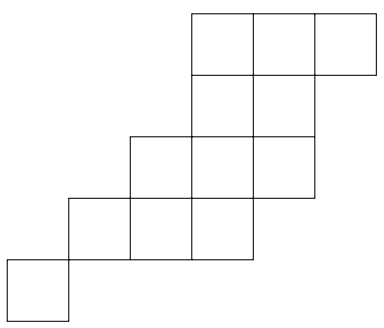

and that we have $\left|\lambda^{(i)}\right|=|\lambda|-j+i-1$ from (2), so we can determine $u_{i}(\lambda)=j$ in terms of $i$ via

$$
u_{i}(\lambda)=|\lambda|-\left|\lambda^{(i)}\right|+i-1 .
$$

Also note that $u_{i}(\lambda)$ weakly decreases as $i$ increases, and so we also obtain

$$
|\lambda|-l(\lambda)=\left|\lambda^{(1)}\right|<\left|\lambda^{(2)}\right|<\cdots, \quad\left|\lambda^{(i)}\right|=|\lambda|+i-1, \quad i>\lambda_{1} .
$$

We also let $\lambda^{(-i)}, i \geq 1$, be the partition whose code is obtained from the code of the partition $\lambda$ by switching the $i$ th $\mathrm{U}$ (from the right) to $\mathrm{R}$. If $\lambda=\left(\lambda_{1}, \lambda_{2}, \ldots\right)$ with $\lambda_{1} \geq \lambda_{2} \ldots \geq 0$ (i.e., $\lambda$ has a finite number, say $n$, of positive parts, and then append an infinite sequence of 0 's), then we immediately have

$$
\lambda^{(-i)}=\left(\lambda_{1}+1, \ldots, \lambda_{i-1}+1, \lambda_{i+1}, \ldots\right) .
$$

Thus we have $\left|\lambda^{(-i)}\right|=|\lambda|-\lambda_{i}+i-1$, and since $\lambda_{i}$ weakly decreases as $i$ increases, we obtain

$$
|\lambda|-\lambda_{1}=\left|\lambda^{(-1)}\right|<\left|\lambda^{(-2)}\right|<\cdots, \quad\left|\lambda^{(-i)}\right|=|\lambda|+i-1, \quad i>l(\lambda) .
$$

Finally, we define skew diagrams. For partitions $\lambda=\left(\lambda_{1}, \ldots, \lambda_{n}\right)$ and $\mu=$ $\left(\mu_{1}, \ldots, \mu_{m}\right)$ with $n \geq m$ and $\lambda_{i} \geq \mu_{i}$ for $i=1, \ldots, m$, the skew diagram $\lambda-\mu$ is the array of unit squares obtained by removing the squares of the diagram of $\mu$ from the diagram of $\lambda$. For example, the skew diagram $(6,5,5,4,1)-(3,3,2,1)$ is given in Fig. 2.

A skew diagram is a horizontal strip if it contains at most one square in every column; it is a vertical strip if it contains at most one square in every row. When the total number of squares in a horizontal (or vertical) strip is equal to $k$, we may refer to this skew diagram as a horizontal (or vertical) $k$-strip.

\section{Codes of partitions and symmetric functions}

\subsection{The algebra of symmetric functions}

We consider symmetric functions in $x_{1}, x_{2}, \ldots$ and refer to [10,14] for the results that we use in this paper. The $i$ th power sum symmetric function is $p_{i}=\sum_{j \geq 1} x_{j}^{i}$ for $i \geq 1$, with $p_{0}:=1$. The $i$ th complete symmetric function $h_{i}$ and the $i$ th elementary 
symmetric function are defined by $\sum_{i \geq 0} h_{i} t^{i}=\prod_{j \geq 1}\left(1-x_{j} t\right)^{-1}$ and $\sum_{i \geq 0} e_{i} t^{i}=$ $\prod_{j \geq 1}\left(1+x_{j} t\right)$, respectively, and are related to the power sums through

$$
\sum_{i \geq 0} h_{i} t^{i}=\exp \sum_{k \geq 1} \frac{p_{k}}{k} t^{k}, \quad \sum_{i \geq 0} e_{i} t^{i}=\exp \sum_{k \geq 1} \frac{p_{k}}{k}(-1)^{k-1} t^{k} .
$$

In the algebra $\Lambda_{\mathbb{Q}}$ of symmetric functions in $x_{1}, x_{2}, \ldots$ over $\mathbb{Q}$, the $p_{i}, i \geq 1$, are algebraically independent, the $h_{i}, i \geq 1$, are algebraically independent, and the $e_{i}$, $i \geq 1$, are algebraically independent. This algebra is graded, by total degree in the underlying indeterminates $x_{1}, x_{2}, \ldots$ Moreover, $\Lambda_{\mathbb{Q}}$ is generated by the $p_{i}$ 's, the $h_{i}$ 's, or the $e_{i}$ 's; so we have

$$
\Lambda_{\mathbb{Q}}=\mathbb{Q}\left[p_{1}, p_{2}, \ldots\right]=\mathbb{Q}\left[h_{1}, h_{2}, \ldots\right]=\mathbb{Q}\left[e_{1}, e_{2}, \ldots\right] .
$$

Now suppose we define quantities $p_{\lambda}, h_{\lambda}, e_{\lambda}$ indexed by the partition $\lambda=$ $\left(\lambda_{1}, \ldots, \lambda_{n}\right)$ multiplicatively, which means that we write $p_{\lambda}=p_{\lambda_{1}} \cdots p_{\lambda_{n}}, h_{\lambda}=$ $h_{\lambda_{1}} \cdots h_{\lambda_{n}}, e_{\lambda}=e_{\lambda_{1}} \cdots e_{\lambda_{n}}$, with the empty product convention $p_{\varepsilon}=h_{\varepsilon}=e_{\varepsilon}=1$. Then of course, regarded as a vector space over $\mathbb{Q}, \Lambda_{\mathbb{Q}}$ has (multiplicative) bases $\left\{p_{\lambda}: \lambda \in \mathcal{P}\right\},\left\{h_{\lambda}: \lambda \in \mathcal{P}\right\}$, and $\left\{e_{\lambda}: \lambda \in \mathcal{P}\right\}$.

Another (nonmultiplicative) basis is $\left\{s_{\lambda}: \lambda \in \mathcal{P}\right\}$, where $s_{\lambda}$ is the Schur function of shape $\lambda$. There are many fascinating aspects about Schur functions, but the one that we shall focus on in this paper is their connection with characters of the symmetric group (we refer to [14] for the results about characters and representations of the symmetric group used in this paper). The conjugacy classes of the symmetric group $\mathfrak{S}_{d}$ on $\{1, \ldots, d\}$ are indexed by the partitions of $d$ (and for partition $\lambda=\left(\lambda_{1}, \ldots, \lambda_{n}\right)$, the conjugacy class $\mathcal{C}_{\lambda}$ consists of all permutations in $\mathfrak{S}_{d}$ whose disjoint cycle lengths give the parts of $\lambda$ in some order). The irreducible representations of $\mathfrak{S}_{d}$ are also indexed by the partitions of $d$. For partitions $\lambda, \mu$ of $d$, let $\chi_{\mu}^{\lambda}$ denote the character of the irreducible representation of $\mathfrak{S}_{d}$ indexed by $\lambda$ and evaluated at any element of the conjugacy class $\mathcal{C}_{\mu}$ (in general, characters are constant on conjugacy classes); we usually refer to $\chi_{\mu}^{\lambda}$ generically as an irreducible character. Then, to change bases of $\Lambda_{\mathbb{Q}}$ between $\left\{p_{\lambda}: \lambda \in \mathcal{P}\right\}$ and $\left\{s_{\lambda}: \lambda \in \mathcal{P}\right\}$, we have

$$
p_{\lambda}=\sum_{\mu \vdash d} \chi_{\lambda}^{\mu} s_{\mu}, \quad s_{\lambda}=\sum_{\mu \vdash d} \frac{\left|\mathcal{C}_{\mu}\right|}{d !} \chi_{\mu}^{\lambda} p_{\mu}, \quad \lambda \vdash d .
$$

The endomorphism $\omega: \Lambda_{\mathbb{Q}} \rightarrow \Lambda_{\mathbb{Q}}$ defined by $\omega\left(e_{n}\right)=h_{n}, n \geq 1$, is an involution on $\Lambda_{\mathbb{Q}}$. For Schur functions, we have

$$
\omega\left(s_{\lambda}\right)=s_{\lambda^{\prime}} .
$$

We have various multiplication rules for Schur functions. For example,

$$
h_{n} s_{\lambda}=\sum_{\mu} s_{\mu}
$$

where the sum is over partitions $\mu$ such that $\mu-\lambda$ is a horizontal $n$-strip. In general, when the involution $\omega$ is applied to an equation for symmetric functions, we call the 
resulting equation the dual. For example, applying $\omega$ to (10), we obtain the dual result

$$
e_{n} s_{\lambda}=\sum_{\mu} s_{\mu}
$$

where the sum is over partitions $\mu$ such that $\mu-\lambda$ is a vertical $n$-strip. (Equations (10) and (11) are often referred to as Pieri rules.)

Let $\langle$,$\rangle be the bilinear form for \Lambda_{\mathbb{Q}}$ defined by

$$
\left\langle s_{\lambda}, s_{\mu}\right\rangle=\delta_{\lambda, \mu}, \quad \lambda, \mu \in \mathcal{P} .
$$

For each symmetric function $f \in \Lambda_{\mathbb{Q}}$, let $f^{\perp}: \Lambda_{\mathbb{Q}} \rightarrow \Lambda_{\mathbb{Q}}$ be the adjoint of multiplication by $f$, so

$$
\left\langle f^{\perp} g_{1}, g_{2}\right\rangle=\left\langle g_{1}, f g_{2}\right\rangle, \quad g_{1}, g_{2} \in \Lambda_{\mathbb{Q}}
$$

Then

$$
p_{n}^{\perp}=n \frac{\partial}{\partial p_{n}}, \quad n \geq 1 .
$$

Moreover, from (10) and (12) we have

$$
h_{n}^{\perp} s_{\lambda}=\sum_{\mu} s_{\mu},
$$

where the sum is over partitions $\mu$ such that $\lambda-\mu$ is a horizontal $n$-strip. Similarly, from (11) and (12) we have

$$
e_{n}^{\perp} s_{\lambda}=\sum_{\mu} s_{\mu},
$$

where the sum is over partitions $\mu$ such that $\lambda-\mu$ is a vertical $n$-strip.

We now consider Bernstein's symmetric function operators $B(t), B_{n}$ defined in (1). Equivalently, from (7) and (13), together with the fact that the partial differential operators $\frac{\partial}{\partial p_{k}}$ commute, we have

$$
B(t):=\sum_{n \in \mathbb{Z}} B_{n} t^{n}:=\sum_{k, m \geq 0}(-1)^{m} t^{k-m} h_{k} e_{m}^{\perp}
$$

(where $B_{n}$ is an operator on $\Lambda_{\mathbb{Q}}$ for each $n \in \mathbb{Z}$ ). Then we also have

$$
B^{\perp}(t)=\sum_{n \in \mathbb{Z}} B_{n}^{\perp} t^{n}=\sum_{k, m \geq 0}(-1)^{m} t^{k-m} e_{m} h_{k}^{\perp},
$$

and we immediately obtain $B_{n}^{\perp}=(-1)^{n} \omega B_{-n} \omega$ (note that this corrects the relation given in [10, p. 96]). In terms of the series $B$ itself, this becomes the operator equation

$$
B^{\perp}(t)=\omega B\left(-t^{-1}\right) \omega,
$$

where $\omega$ applied to a series in $t$ acts linearly on the coefficients of each monomial in $t$. 
Fig. 3 Partitions $\lambda$ and $\mu$ with $\lambda$-ambiguous squares
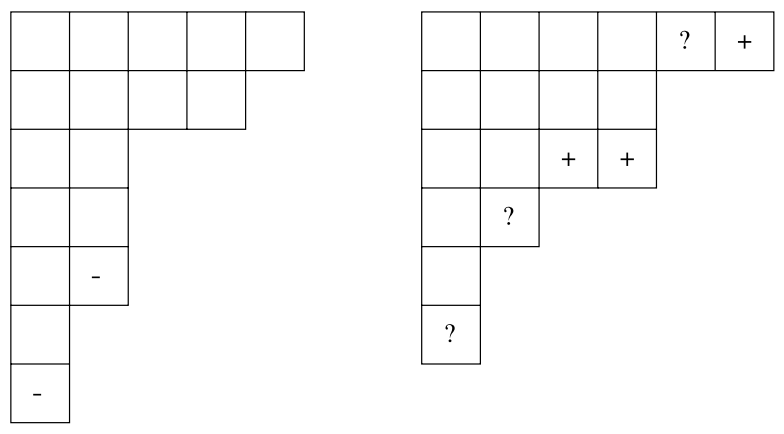

\subsection{A combinatorial treatment of Bernstein's operator}

We define $\mathcal{R}_{\lambda, \mu}$ to be the set of partitions $v$ such that $\lambda-v$ is a vertical strip and $\mu-v$ is a horizontal strip. Let

$$
R_{\lambda, \mu}=\sum_{\nu \in \mathcal{R}_{\lambda, \mu}}(-1)^{|\lambda|-|\nu|},
$$

which is 0 when the set $\mathcal{R}_{\lambda, \mu}$ is empty. This sum arises naturally in the action of $B(t)$ on a Schur function $s_{\lambda}$, as given in the following result.

Proposition 3.1 For any partition $\lambda$, we have

$$
B(t) s_{\lambda}=\sum_{\mu \in \mathcal{P}} R_{\lambda, \mu} t^{|\mu|-|\lambda|} s_{\mu} .
$$

Proof The result follows immediately from (16), (10), and (15).

Now we consider the structure of the set $\mathcal{R}_{\lambda, \mu}$, beginning with the example given in Fig. 3, where the diagrams of $\lambda=(5,4,2,2,2,1,1)$ and $\mu=(6,4,4,2,1,1)$ are given on the left and right of the diagram, respectively. There are three classes of squares in the union of the diagrams for $\lambda$ and $\mu$ that we shall consider when $\mathcal{R}_{\lambda, \mu}$ is nonempty:

- The squares of $\mu$ that are not contained in $\lambda$. These squares are necessarily bottommost in their column of $\mu$. None of these is contained in any $v$ in $\mathcal{R}_{\lambda, \mu}$ (in Fig. 3, these squares contain " + "). Such squares are contained in the horizontal strip that is added in the multiplication by an $h_{k}$.

- The squares of $\lambda$ that are not contained in $\mu$. These squares are necessarily rightmost in their row of $\lambda$. None of these squares is contained in any $v$ in $\mathcal{R}_{\lambda, \mu}$ (in Fig. 3, these squares contain “-”). Such squares are contained in the vertical strip that is deleted in the application of $e_{m}^{\perp}$.

- The squares that are contained in both $\lambda$ and $\mu$. These squares are necessarily both rightmost in their row of $\lambda$, and bottommost in their column of $\mu$. Each of these is contained in some of the $v$ in $\mathcal{R}_{\lambda, \mu}$, but not others (in Fig. 3, the $\lambda$-ambiguous squares of $\mu$ contain “?”). Such squares may have been contained in both a deleted 
vertical strip and an added horizontal strip or neither. We call the squares in $\mu$ with this property $\lambda$-ambiguous.

Lemma 3.2 If $\mu$ has any $\lambda$-ambiguous squares, then

$$
R_{\lambda, \mu}=0 \text {. }
$$

Proof If $\mu$ has any $\lambda$-ambiguous squares, let $c$ be the rightmost of these (there is at most one $\lambda$-ambiguous square in any column of $\mu$, since it can only occur as the bottommost element of a column). Define the mapping

$$
\phi: \mathcal{R}_{\lambda, \mu} \rightarrow \mathcal{R}_{\lambda, \mu}: v \mapsto v^{\prime}
$$

as follows: if $v$ contains $c$, then $v^{\prime}$ is obtained by removing $c$ from $v$; if $v$ does not contain $c$, then $v^{\prime}$ is obtained by adding $c$ to $v$. This is well defined for the following reasons: since $c$ is rightmost in its row of $\lambda$ and bottommost in its column of $\mu$, every square of $\lambda$ in the same column as $c$ and below $c$ must belong to the vertical strip $\lambda-v$ (and no other squares in this column can belong to this vertical strip), so $\lambda-v^{\prime}$ is a vertical strip whether $v$ contains $c$ or not; also, every square of $\mu$ in the same row as $c$ and to the right of $c$ must belong to the horizontal strip $\mu-v$ (and no other squares in this row can belong to this horizontal strip), so $\mu-v^{\prime}$ is a horizontal strip whether $v$ contains $c$ or not).

Clearly $\phi$ is an involution on $\mathcal{R}_{\lambda, \mu}$, so it is a bijection, and thus we have

$$
R_{\lambda, \mu}=\sum_{\nu \in \mathcal{R}_{\lambda, \mu}}(-1)^{|\lambda|-|\nu|}=-\sum_{\nu^{\prime} \in \mathcal{R}_{\lambda, \mu}}(-1)^{|\lambda|-\left|\nu^{\prime}\right|}=-R_{\lambda, \mu},
$$

where, for the second equality, we have changed summation variables to $\phi(v)=v^{\prime}$. The result follows immediately. (In the context of (20), $\phi$ is often referred to as a sign-reversing involution.)

If $\mathcal{R}_{\lambda, \mu}$ is nonempty and $\mu$ has no $\lambda$-ambiguous squares, we call $\mu$ a $\lambda$-survivor. Note that in this case there is a unique $\nu$ in $\mathcal{R}_{\lambda, \mu}$; the terminology is chosen since $\mu$ "survives" the involution in Lemma 3.2.

Proposition 3.3 In a $\lambda$-survivor $\mu$ :

(a) If the rightmost square of row $i$ of $\lambda$ is not contained in $\mu$, then the rightmost square of row $i-1$ of $\lambda$ is not contained in $\mu$.

(b) If the bottommost square of column i of $\mu$ is not contained in $\lambda$, then the bottommost square of column $i-1$ of $\mu$ is not contained in $\lambda$.

Proof For part (a), if the rightmost square of row $i$ of $\lambda$ is not contained in $\mu$ and the rightmost square of row $i-1$ of $\lambda$ is contained in $\mu$, then the latter must be bottommost in its column of $\mu$. But that makes it a $\lambda$-ambiguous square, impossible in a $\lambda$-survivor. For (b), if the bottommost square of column $i$ of $\mu$ is not contained in $\lambda$ and the bottommost square of column $i-1$ of $\mu$ is contained in $\lambda$, then the latter is by definition a $\lambda$-ambiguous square, impossible in a $\lambda$-survivor. 


\subsection{The main result}

Now we are able to determine explicitly the action of $B(t)$ on a single Schur function $s_{\lambda}$. The following is our main result.

Theorem 3.4 For any partition $\lambda$, we have

$$
B(t) s_{\lambda}=\sum_{i \geq 1}(-1)^{|\lambda|-\left|\lambda^{(i)}\right|+i-1} t^{\left|\lambda^{(i)}\right|-|\lambda|} s_{\lambda^{(i)}} .
$$

Proof From Proposition 3.1 and Lemma 3.2 we have

$$
B(t) s_{\lambda}=\sum_{\mu} R_{\lambda, \mu} t^{|\mu|-|\lambda|} s_{\mu},
$$

where the summation is over all $\lambda$-survivors $\mu$. Now we characterize the $\lambda$-survivors. Suppose $\lambda=\left(\lambda_{1}, \ldots, \lambda_{n}\right)$, where $\lambda_{1} \geq \cdots \geq \lambda_{n} \geq 1$, and let $\lambda_{0}=\infty, \lambda_{n+1}=0$. Then in a $\lambda$-survivor $\mu$, from Proposition 3.3(a), the rightmost cells in rows $1, \ldots, j$ of $\lambda$ are not contained in $\mu$, and the rightmost cells of rows $j+1, \ldots, n$ are contained in $\mu$ for some $j=0, \ldots, n$ with $\lambda_{j}>\lambda_{j+1}$. But, in order to avoid the bottommost cell of column $\lambda_{j+1}$ in $\mu$ being $\lambda$-ambiguous, the bottommost cell of column $\lambda_{j+1}$ in $\mu$ cannot be contained in $\lambda$. Thus we conclude from Proposition 3.3(b) that the bottommost cells in columns $1, \ldots, \lambda_{j+1}$ of $\mu$ are not contained in $\lambda$. Also, the bottommost squares in columns $\lambda_{j+1}, \ldots, i-1$ of $\mu$ are not contained in $\lambda$ for some $\lambda_{j+1}<i \leq \lambda_{j}$. Finally, for each $i \geq 1$, there exists a choice of $j=0, \ldots, n$ for which $\lambda_{j+1}<i \leq \lambda_{j}$, so the $\lambda$-survivor $\mu$ described above exists for each $i \geq 1$. This partition $\mu$ is obtained from $\lambda$ by deleting the column strip consisting of the rightmost squares in rows $1, \ldots, j$ and adding the horizontal strip consisting of the bottommost cells in columns $1, \ldots, i-1$. This gives

$$
\mu=\left(\lambda_{1}-1, \ldots, \lambda_{j}-1, i-1, \lambda_{j+1}, \ldots, \lambda_{n}\right)=\lambda^{(i)},
$$

where the second equality is from (2), and where $j=u_{i}(\lambda)$. But we have $R_{\lambda, \lambda^{(i)}}=$ $(-1)^{j}$, and the result follows from (3) and (21).

Note that the right-hand side of the above result is a Laurent series in $t$ for each $\lambda$, with minimum power of $t$ given by $t^{\left|\lambda^{(1)}\right|-|\lambda|}=t^{-l(\lambda)}$.

The following pair of dual corollaries to our main result will be particularly convenient for dealing with the KP hierarchy.

Corollary 3.5 For scalars $a_{\lambda}, \lambda \in \mathcal{P}$, we have

$$
B(t) \sum_{\lambda \in \mathcal{P}} a_{\lambda} s_{\lambda}=\sum_{\beta \in \mathcal{P}} s_{\beta} \sum_{k \geq 1}(-1)^{k-1} t^{|\beta|-\left|\beta^{(-k)}\right|} a_{\beta^{(-k)}} .
$$

Proof From Theorem 3.4 and (3) we immediately obtain

$$
B(t) \sum_{\lambda \in \mathcal{P}} a_{\lambda} s_{\lambda}=\sum_{\lambda \in \mathcal{P}} a_{\lambda} \sum_{i \geq 1}(-1)^{u_{i}(\lambda)} t^{\left|\lambda^{(i)}\right|-|\lambda|} s_{\lambda^{(i)}} .
$$


Now from the code description it is immediate that $\beta=\lambda^{(i)}$ is equivalent to $\lambda=$ $\beta^{(-k)}$, where $k=u_{i}(\lambda)+1$. The result follows immediately by changing summation variables in (22) from $\lambda \in \mathcal{P}, i \geq 1$, to $\beta \in \mathcal{P}, k \geq 1$.

Corollary 3.6 For scalars $a_{\lambda}, \lambda \in \mathcal{P}$, we have

$$
B^{\perp}\left(t^{-1}\right) \sum_{\lambda \in \mathcal{P}} a_{\lambda} s_{\lambda}=\sum_{\alpha \in \mathcal{P}} s_{\alpha} \sum_{m \geq 1}(-1)^{|\alpha|-\left|\alpha^{(m)}\right|+m-1} t^{|\alpha|-\left|\alpha^{(m)}\right|} a_{\alpha^{(m)}} .
$$

Proof From Theorem 3.4, (18), and (9) we obtain

$$
B^{\perp}\left(t^{-1}\right) \sum_{\lambda \in \mathcal{P}} a_{\lambda} s_{\lambda}=\sum_{\lambda \in \mathcal{P}} a_{\lambda} \omega B(-t) s_{\lambda^{\prime}}=\sum_{\lambda \in \mathcal{P}} a_{\lambda} \sum_{i \geq 1}(-1)^{i-1} t^{\left|\left(\lambda^{\prime}\right)^{(i)}\right|-\left|\lambda^{\prime}\right|} s_{\left(\left(\lambda^{\prime}\right)^{(i)}\right)^{\prime}}
$$

But from the code description it is immediate that $\left(\lambda^{\prime}\right)^{(i)}=\left(\lambda^{(-i)}\right)^{\prime}$, and since $\left|\mu^{\prime}\right|=$ $|\mu|$ for any partition $\mu$, we can simplify the double summation above to obtain

$$
B^{\perp}\left(t^{-1}\right) \sum_{\lambda \in \mathcal{P}} a_{\lambda} s_{\lambda}=\sum_{\lambda \in \mathcal{P}} a_{\lambda} \sum_{i \geq 1}(-1)^{i-1} t^{\left|\lambda^{(-i)}\right|-|\lambda|} s_{\lambda(-i)} .
$$

As in the proof of Corollary 3.5, we have that $\alpha=\lambda^{(-i)}$ is equivalent to $\lambda=\alpha^{(m)}$, where $i=u_{m}(\alpha)+1$. The result now follows immediately by changing summation variables in (22) from $\lambda \in \mathcal{P}, i \geq 1$, to $\alpha \in \mathcal{P}, m \geq 1$, and applying (3) to evaluate $u_{m}(\alpha)$ (which is the exponent of $(-1)$ when the summation is expressed in terms of $\alpha, m)$.

Among the results in [10] and [16] for Bernstein's operators is

$$
B_{\lambda_{1}} \cdots B_{\lambda_{n}} 1=s_{\lambda}
$$

where $\lambda=\left(\lambda_{1}, \ldots, \lambda_{n}\right)$. This result follows immediately from Theorem 3.4 , together with (4). To compose $B_{i}$ when they are not ordered as in this result, one simply uses the result that $B_{i} B_{j}=-B_{j-1} B_{i+1}$, which follows routinely from Theorem 3.4 and considering what happens when two right-steps are switched to up-steps in the two possible orders.

The Bernstein operators were also used in [3] to give an alternate proof of the Littlewood-Richardson rule. A similar operator was used in [4] to give a formula analogous to (24) for Schur Q-functions. See also [5, Chap. 7] for more on the Schur Q-function variant.

\section{Codes of partitions and the Plücker relations}

We consider a set $a_{\mathcal{P}}=\left\{a_{\lambda}: \lambda \in \mathcal{P}\right\}$ of scalars indexed by the set $\mathcal{P}$ of partitions. Then the Plücker relations for $a_{\mathcal{P}}$ are given by the following system of simultaneous quadratic equations: for all $m \geq 1$ and $\alpha=\left(\alpha_{1}, \ldots, \alpha_{m-1}\right), \beta=\left(\beta_{1}, \ldots, \beta_{m+1}\right) \in \mathcal{P}$ 
with $l(\alpha) \leq m-1, l(\beta) \leq m+1$ (which means that $\alpha_{i}=0$ for $i>l(\alpha)$ and $\beta_{i}=0$ for $i>l(\beta))$, we have

$$
\begin{aligned}
& \sum_{k=0}^{m}(-1)^{k-m+1+\ell} a_{\left(\alpha_{1}-1, \ldots, \alpha_{\ell}-1, \beta_{k+1}-k+\ell+1, \alpha_{\ell+1}, \ldots, \alpha_{m-1}\right)} \\
& \quad \times a_{\left(\beta_{1}+1, \ldots, \beta_{k}+1, \beta_{k+2}, \ldots, \beta_{m+1}\right)}=0
\end{aligned}
$$

where $\ell=\ell(k)$ is chosen so that $0 \leq \ell \leq m-1$ and

$$
\alpha_{\ell}-1 \geq \beta_{k+1}-k+\ell+1 \geq \alpha_{\ell+1},
$$

with the convention that $\alpha_{0}=\infty, \alpha_{m}=-\infty$, and so that $\beta_{k+1}-k+\ell+1 \geq 0$ (if there is no such choice of $\ell$, then the term in the summation indexed by $k$ is identically 0 ). Note that, for each choice of $k$, then $\ell$ (if it exists) is unique; to see this, let $\gamma_{i}=\alpha_{i}-i$, $i=0, \ldots, m$. Then $\infty=\gamma_{0}>\gamma_{1}>\cdots>\gamma_{m}=-\infty$, and so for all real numbers $x$, there is a unique $0 \leq i \leq m-1$ such that $\gamma_{i}>x \geq \gamma_{i+1}$. Then, rewriting (26), $\ell$ is the unique choice of $i$ such that (more restrictively, there may not be such an $i$ ) $\gamma_{i}-2 \geq x \geq \gamma_{i+1}$ with $x=\beta_{k+1}-k$.

The presentation of the Plücker relations given in (25) above is referred to as "classical" by Fulton [1, p. 133]. In this presentation, each equation is a quadratic alternating summation corresponding to an ordered pair of partitions. Each term in the alternating summation arises from removing a single part from the second partition and inserting it into the first partition, with some appropriate shift in the remaining parts of both partitions. In our next result, we give a different presentation of the Plücker relations, which is more symmetrical in its form, using the notation developed in Sect. 2 for codes of partitions.

Theorem 4.1 The Plücker relations for $a_{\mathcal{P}}$ are given by the following system of simultaneous quadratic equations: for all $\alpha, \beta \in \mathcal{P}$, we have

$$
\sum_{\substack{i, j \geq 1 \\(-j)|=| \alpha|+| \beta \mid+1}}(-1)^{|\alpha|-\left|\alpha^{(i)}\right|+i+j} a_{\alpha^{(i)}} \cdot a_{\beta^{(-j)}}=0 .
$$

Proof In the Plücker relations, (25) is satisfied for each $(m, \alpha, \beta)$ for $m \geq 1$ and $\alpha, \beta \in \mathcal{P}$ with $l(\alpha) \leq m-1, l(\beta) \leq m+1$. Now multiply $(25)$ by $(-1)^{m-1}$, to get an equation we'll call $(25)^{\prime}$, and consider $(25)^{\prime}$ for $(m+1, \alpha, \beta)$, where we have $\alpha_{m}=\beta_{m+2}=0$. Then on the left-hand side, the term indexed by $k=m+1$ in the latter equation is identically 0 , since there is no possible choice of $\ell$ (to see this, we must have $\beta_{k+1}-k+\ell+1 \geq 0$, so $\ell \geq m$, and since $0 \leq \ell \leq m$, we must uniquely have $\ell=m$; but then we have $\alpha_{\ell}-1=-1<0=\beta_{k+1}-k+\ell+1$, contradicting (26)). Thus $(25)^{\prime}$ for $(m, \alpha, \beta)$ is identical to $(25)^{\prime}$ for $(m+1, \alpha, \beta)$, so there is the following single equation for each $\alpha=\left(\alpha_{1}, \alpha_{2}, \ldots\right), \beta=\left(\beta_{1}, \beta_{2}, \ldots\right) \in \mathcal{P}$ (which means that $\alpha_{i}=0$ for $i>l(\alpha)$ and $\beta_{i}=0$ for $\left.i>l(\beta)\right)$ :

$$
\sum_{k \geq 0}(-1)^{k+\ell} a_{\left(\alpha_{1}-1, \ldots, \alpha_{\ell}-1, \beta_{k+1}-k+\ell+1, \alpha_{\ell+1}, \ldots\right)} \cdot a_{\left(\beta_{1}+1, \ldots, \beta_{k}+1, \beta_{k+2}, \ldots\right)}=0,
$$


where $\ell=\ell(k)$ is chosen so that

$$
\alpha_{\ell}-1 \geq \beta_{k+1}-k+\ell+1 \geq \alpha_{\ell+1},
$$

with the convention that $\alpha_{0}=\infty$, and so that $\beta_{k+1}-k+\ell+1 \geq 0$. But, by (2) and (5), (27) becomes

$$
\sum_{k \geq 0}(-1)^{k+\ell} a_{\alpha^{\left(\beta_{k+1}-k+\ell+2\right)}} \cdot a_{\beta^{(-k-1)}}=0 .
$$

Finally, note that

$$
\left|\alpha^{\left(\beta_{k+1}-k+\ell+2\right)}\right|+\left|\beta^{(-k-1)}\right|=|\alpha|+|\beta|+1,
$$

and the result follows from (3), (4), and (6).

To fix ideas, we now give a few examples of Plücker relations. These examples illustrate that there are redundant equations in the Plücker relations.

Example 4.2 For $\alpha=\beta=(1)$, we obtain $\alpha^{(1)}=\varepsilon, \alpha^{(2)}=(1,1), \alpha^{(3)}=(2,1)$, and $\beta^{(-1)}=\varepsilon, \beta^{(-2)}=(2), \beta^{(-3)}=(2,1)$, so the corresponding quadratic equation is

$$
-a_{\varepsilon} \cdot a_{(2,1)}+a_{(2,1)} \cdot a_{\varepsilon}=0 .
$$

But the left-hand side of this equation is identically 0 , so the equation is redundant.

For $\alpha=\varepsilon, \beta=(1,1,1)$, we obtain $\alpha^{(1)}=\varepsilon, \alpha^{(2)}=(1), \alpha^{(3)}=(2), \alpha^{(4)}=(3)$, and $\beta^{(-1)}=(1,1), \beta^{(-2)}=(2,1), \beta^{(-3)}=(2,2)$, so the corresponding quadratic equation is

$$
a_{\varepsilon} \cdot a_{(2,2)}-a_{(1)} \cdot a_{(2,1)}+a_{(2)} \cdot a_{(1,1)}=0 .
$$

For $\alpha=(2), \beta=(1)$, we obtain $\alpha^{(1)}=(1), \alpha^{(2)}=(1,1), \alpha^{(3)}=(2,2)$, and $\beta^{(-1)}=$ $\varepsilon, \beta^{(-2)}=(2), \beta^{(-3)}=(2,1)$, so the corresponding quadratic equation is

$$
-a_{(1)} \cdot a_{(2,1)}+a_{(1,1)} \cdot a_{(2)}+a_{(2,2)} \cdot a_{\varepsilon}=0,
$$

which is the same equation as (28).

\section{$5 \tau$-functions for the KP hierarchy and the Plücker relations}

We now make use of the preceding results to show that solutions to the KP hierarchy admit a combinatorial description in terms of power series whose Schur function coefficients satisfy the Plücker relations. This result is well known in the literature, however, our presentation is new.

There are a number of equivalent descriptions of the KP hierarchy, as is well described in [11]. The one that we shall start with in this paper involves the so-called $\tau$-function of the hierarchy and two independent sets of indeterminates $p_{1}, p_{2}, \ldots$ 
and $\widehat{p}_{1}, \widehat{p}_{2}, \ldots$ A power series is said to be a $\tau$-function for the KP hierarchy if and only if it satisfies the bilinear equation

$$
\left[t^{-1}\right] \exp \left(\sum_{k \geq 1} \frac{t^{k}}{k}\left(p_{k}-\widehat{p}_{k}\right)\right) \exp \left(-\sum_{k \geq 1} t^{-k}\left(\frac{\partial}{\partial p_{k}}-\frac{\partial}{\partial \widehat{p}_{k}}\right)\right) \tau(\mathbf{p}) \tau(\widehat{\mathbf{p}})=0,
$$

where $\mathbf{p}=\left(p_{1}, p_{2}, \ldots\right), \widehat{\mathbf{p}}=\left(\widehat{p}_{1}, \widehat{p}_{2}, \ldots\right)$, and the notation $[A] B$ means the coefficient of $A$ in $B$.

In considering (29), we regard $p_{1}, p_{2}, \ldots$ as the power sum symmetric functions of an underlying set of variables $x_{1}, x_{2}, \ldots$, as in Sect. 3. Also, we regard $\widehat{p}_{1}, \widehat{p}_{2}, \ldots$ as the power sum symmetric functions of another set of variables, algebraically independent of $x_{1}, x_{2}, \ldots$ (one could write this other set of variables as $\widehat{x}_{1}, \widehat{x}_{2}, \ldots$, say, but the precise names of these variables are irrelevant, since no further explicit mention of either $x_{1}, x_{2}, \ldots$ or $\widehat{x}_{1}, \widehat{x}_{2}, \ldots$ will be made). We shall use the notation $\widehat{e}_{i}, \widehat{h}_{i}, \widehat{s}_{\lambda}$, $\widehat{B}(t)$ to denote the symmetric functions in this other set of variables corresponding to $e_{i}, h_{i}, s_{\lambda}, B(t)$ in $x_{1}, x_{2}, \ldots$

The following result follows immediately by taking this symmetric function view of (29). This view has appeared in a number of earlier works (see, e.g., [6]), but the results from symmetric functions that have been applied in these works have been different than ours.

Proposition 5.1 A power series is a $\tau$-function for the KP hierarchy if and only if it satisfies

$$
\left[t^{-1}\right](B(t) \tau(\mathbf{p})) \cdot\left(\widehat{B}^{\perp}\left(t^{-1}\right) \tau(\widehat{\mathbf{p}})\right)=0 .
$$

Proof The result follows immediately from (29), together with (1) and (13).

Next we give a proof of the connection between Schur function coefficients of a $\tau$-function for the KP hierarchy, and the Plücker relations. Our proof is immediate from Corollaries 3.5 and 3.6.

Theorem 5.2 (See, e.g., [11, p. 90, (10.3)]) Let $a_{\lambda}$ be the coefficient of the Schur function of shape $\lambda$ in a power series, for $\lambda \in \mathcal{P}$. Then the power series is a $\tau$-function for the KP hierarchy if and only if $a_{\mathcal{P}}=\left\{a_{\lambda}: \lambda \in \mathcal{P}\right\}$ satisfies the Plücker relations.

Proof We are given $\tau(\mathbf{p})=\sum_{\lambda \in \mathcal{P}} a_{\lambda} s_{\lambda}$ and $\tau(\widehat{\mathbf{p}})=\sum_{\lambda \in \mathcal{P}} a_{\lambda} \widehat{s_{\lambda}}$. Then, from (30), it is necessary and sufficient that $a_{\mathcal{P}}$ satisfies $S(\mathbf{p}, \widehat{\mathbf{p}})=0$, where

$$
S(\mathbf{p}, \widehat{\mathbf{p}})=\left[t^{-1}\right]\left(B(t) \sum_{\lambda \in \mathcal{P}} a_{\lambda} s_{\lambda}\right) \cdot\left(\widehat{B}^{\perp}\left(t^{-1}\right) \sum_{\mu \in \mathcal{P}} a_{\mu} \widehat{s}_{\mu}\right) .
$$

Now, from Corollaries 3.5 and 3.6 we immediately obtain

$$
S(\mathbf{p}, \widehat{\mathbf{p}})=\sum_{\beta, \alpha \in \mathcal{P}} s_{\beta} \widehat{s}_{\alpha} \sum_{\substack{m, k \geq 1 \\\left|\alpha^{(m)}\right|+\left|\beta^{(-k)}\right|=|\alpha|+|\beta|+1}}(-1)^{|\alpha|-\left|\alpha^{(m)}\right|+m+k} a_{\alpha^{(m)}} \cdot a_{\beta^{(-k)}} .
$$


But $S(\mathbf{p}, \widehat{\mathbf{p}})=0$ if and only if $\left[s_{\beta} \widehat{s}_{\alpha}\right] S(\mathbf{p}, \widehat{\mathbf{p}})=0$ for all $\beta, \alpha \in \mathcal{P}$, since the Schur functions form a basis for symmetric functions, and the result follows immediately from Theorem 4.1.

Often the KP hierarchy is written as a system of simultaneous quadratic partial differential equations for $\tau$. In the next result, we apply Theorem 5.2 and the methods of symmetric functions to obtain such a system of partial differential equations, with one equation corresponding to each quadratic equation in the Plücker relations. The result is well known, but we include a simple proof for completeness.

Theorem 5.3 (See, e.g., [11, p. 92, Lemma 10.2]) The power series $\tau(\mathbf{p})$ is a $\tau$ function for the KP hierarchy if and only if the following partial differential equation is satisfied for each pair of partitions $\alpha$ and $\beta$ :

$$
\sum_{\substack{i, j \geq 1 \\\left|\alpha^{(i)}\right|+\left|\beta^{(-j)}\right|=|\alpha|+|\beta|+1}}(-1)^{|\alpha|-\left|\alpha^{(i)}\right|+i+j}\left(s_{\alpha^{(i)}}\left(\mathbf{p}^{\perp}\right) \tau(\mathbf{p})\right) \cdot\left(s_{\beta^{(-j)}}\left(\mathbf{p}^{\perp}\right) \tau(\mathbf{p})\right)=0
$$

(where, e.g., $s_{\lambda}\left(\mathbf{p}^{\perp}\right)$ is interpreted as the partial differential operator obtained by substituting $p_{n}^{\perp}$ for $p_{n}$ in (8) for each $n \geq 1$ and using (13).)

Proof Let $\mathbf{q}=\left(q_{1}, q_{2}, \ldots\right)$, where the $q_{i}$ are independent of the $p_{j}$ and $\widehat{p}_{k}$. We begin the proof by proving that (I) $\tau$ satisfies (30) if and only if (II) $\tau$ satisfies

$$
\left[t^{-1}\right](B(t) \tau(\mathbf{p}+\mathbf{q})) \cdot\left(\widehat{B}^{\perp}\left(t^{-1}\right) \tau(\widehat{\mathbf{p}}+\mathbf{q})\right)=0
$$

for all $\mathbf{q}$.

It is easy to see that (II) implies (I) by setting $\mathbf{q}=\mathbf{0}$ (where $\mathbf{0}=(0,0, \ldots)$ ).

To prove that (I) implies (II), define the operator $\Theta(\mathbf{p})=\exp \left(\sum_{k \geq 1} q_{k} \frac{\partial}{\partial p_{k}}\right)$. Using the multivariate Taylor series expansion of an arbitrary formal power series $f(\mathbf{p})$, we see that

$$
f(\mathbf{p}+\mathbf{q})=\Theta(\mathbf{p}) f(\mathbf{p}) .
$$

Also define $\Gamma(\mathbf{p})=\exp \left(\sum_{i \geq 1} \frac{t^{i}}{i} p_{i}\right)$ and $\Upsilon(\mathbf{p})=\exp \left(-\sum_{j \geq 1} t^{-j} \frac{\partial}{\partial p_{j}}\right)$, so $B(t)=$ $\Gamma(\mathbf{p}) \Upsilon(\mathbf{p})$. Then from (32) and the trivial fact that $\Upsilon(\mathbf{p})$ commutes with $\Theta(\mathbf{p})$ we have

$$
B(t) \tau(\mathbf{p}+\mathbf{q})=\Gamma(\mathbf{p}) \Upsilon(\mathbf{p}) \Theta(\mathbf{p}) \tau(\mathbf{p})=\Gamma(\mathbf{p}) \Theta(\mathbf{p}) \Upsilon(\mathbf{p}) \tau(\mathbf{p}) .
$$

But using (32) again, we have the operator identity

$$
\Theta(\mathbf{p}) \Gamma(\mathbf{p})=\Gamma(\mathbf{p}+\mathbf{q}) \Theta(\mathbf{p})=\Gamma(\mathbf{q}) \Gamma(\mathbf{p}) \Theta(\mathbf{p}),
$$

and combining these expressions and the fact that $\Gamma(\mathbf{q})^{-1}=\Gamma(-\mathbf{q})$ gives

$$
B(t) \tau(\mathbf{p}+\mathbf{q})=\Gamma(-\mathbf{q}) \Theta(\mathbf{p}) B(t) \tau(\mathbf{p}) .
$$

Similarly we have $\widehat{B}^{\perp}\left(t^{-1}\right)=\Gamma(-\widehat{\mathbf{p}}) \Upsilon(-\widehat{\mathbf{p}})$ and then obtain

$$
\widehat{B}^{\perp}\left(t^{-1}\right) \tau(\widehat{\mathbf{p}}+\mathbf{q})=\Gamma(\mathbf{q}) \Theta(\widehat{\mathbf{p}}) \widehat{B}^{\perp}\left(t^{-1}\right) \tau(\widehat{\mathbf{p}}) .
$$


Multiplying these two expressions together, we find that (31) becomes

$$
\Theta(\mathbf{p}) \Theta(\widehat{\mathbf{p}})\left[t^{-1}\right](B(t) \tau(\mathbf{p})) \cdot\left(\widehat{B}^{\perp}\left(t^{-1}\right) \tau(\widehat{\mathbf{p}})\right)=0,
$$

since $\Gamma(-\mathbf{q}) \Gamma(\mathbf{q})=1$, and $\Theta(\mathbf{p}), \Theta(\widehat{\mathbf{p}})$ are independent of $t$. We conclude that (I) implies (II).

Finally, in order to apply Theorem 5.2, we determine the coefficient of the Schur function of shape $\lambda$. This gives

$$
\begin{aligned}
{\left[s_{\lambda}(\mathbf{p})\right] \tau(\mathbf{p}+\mathbf{q}) } & =\left\langle s_{\lambda}(\mathbf{p}), \tau(\mathbf{p}+\mathbf{q})\right\rangle=\left\langle 1, s_{\lambda}\left(\mathbf{p}^{\perp}\right) \tau(\mathbf{p}+\mathbf{q})\right\rangle \\
& =\left.s_{\lambda}\left(\mathbf{p}^{\perp}\right) \tau(\mathbf{p}+\mathbf{q})\right|_{\mathbf{p}=\mathbf{0}}=s_{\lambda}\left(\mathbf{q}^{\perp}\right) \tau(\mathbf{q}),
\end{aligned}
$$

and the result then follows from Theorem 5.2, replacing $\mathbf{q}$ by $\mathbf{p}$.

As an example of Theorem 5.3, we now give one of the quadratic partial differential equations for a $\tau$-function.

Example 5.4 Consider the Plücker equation (28). Now we have

$$
\begin{aligned}
& s_{\varepsilon}=1, \quad s_{(1)}=p_{1}, \quad s_{(2)}=\frac{1}{2}\left(p_{1}^{2}+p_{2}\right), \quad s_{(1,1)}=\frac{1}{2}\left(p_{1}^{2}-p_{2}\right), \\
& s_{(2,1)}=\frac{1}{3}\left(p_{1}^{3}-p_{3}\right), \quad s_{(2,2)}=\frac{1}{12}\left(p_{1}^{4}-4 p_{1} p_{3}+3 p_{2}^{2}\right),
\end{aligned}
$$

so from Theorem 5.3, the partial differential equation for $\tau$ that corresponds to (28) is given by

$$
\frac{1}{12} \tau\left(\tau_{1111}-12 \tau_{13}+12 \tau_{22}\right)-\frac{1}{3} \tau_{1}\left(\tau_{111}-3 \tau_{3}\right)+\frac{1}{4}\left(\tau_{11}+2 \tau_{2}\right)\left(\tau_{11}-2 \tau_{2}\right)=0,
$$

where we use $\tau_{i j k}$ to denote $\frac{\partial}{\partial p_{i}} \frac{\partial}{\partial p_{j}} \frac{\partial}{\partial p_{k}} \tau$, etc.

Often, in the literature of integrable systems, the series $F=\log \tau$ is used instead of $\tau$ itself. This series $F$ is often referred to as a solution to the KP hierarchy, where the "KP hierarchy" in this context refers to a system of simultaneous partial differential equations for $F$. Of course, the system of partial differential equations for $\tau$ given in Theorem 5.3 becomes an equivalent system of partial differential equations for $F$ by substituting $\tau=\exp F$ into the equations of Theorem 5.3 and then multiplying the equation by $\exp (-2 F)$. For example, when we apply this to (33), we obtain the equation

$$
\frac{1}{12} F_{1111}-F_{13}+F_{22}+\frac{1}{2} F_{11}^{2}=0
$$

which is often referred to as "the KP equation."

Acknowledgements We thank Kevin Purbhoo, Richard Stanley, and Ravi Vakil for helpful suggestions. 


\section{References}

1. Fulton, W.: Young Tableaux: With Applications to Representation Theory and Geometry. Cambridge University Press, Cambridge (1997)

2. Goulden, I.P., Jackson, D.M.: The KP hierarchy, branched covers, and triangulations. Adv. Math. 219, 932-951 (2008)

3. Hoffman, P.N.: Littlewood-Richardson without algorithmically defined bijections. Séminaire Lotharingien de Combinatoire, B20k (1988), 6 pp

4. Hoffman, P.N.: A Bernstein-type formula for projective representations of $S_{n}$ and $A_{n}$. Adv. Math. 74, 135-143 (1989)

5. Hoffman, P.N., Humphreys, J.F.: Projective Representations of the Symmetric Groups. $Q$-Functions and Shifted Tableaux. Oxford Mathematical Monographs. Oxford Science Publications. Clarendon/Oxford University Press, New York (1992), xiv+304 pp

6. Jarvis, P.D., Yung, C.M.: Symmetric functions and the KP and BKP hierarchies. J. Phys. A 26, 59055922 (1993)

7. Kazarian, M.: KP hierarchy for Hodge integrals. Adv. Math. 221, 1-21 (2009)

8. Kazarian, M., Lando, S.: An algebro-geometric proof of Witten's conjecture. J. Am. Math. Soc. 20, 1079-1089 (2007)

9. Kontsevich, M.: Intersection theory on the moduli space of curves and the matrix Airy function. Commun. Math. Phys. 147, 1-23 (1992)

10. Macdonald, I.G.: Symmetric Functions and Hall Polynomials, 2nd edn. Oxford University Press, London (1995)

11. Miwa, T., Jimbo, M., Date, E.: Solitons: Differential Equations, Symmetries and Infinite Dimensional Algebras. Cambridge University Press, Cambridge (2000)

12. Okounkov, A.: Toda equations for Hurwitz numbers. Math. Res. Lett. 7, 447-453 (2000)

13. Pandharipande, R.: The Toda equations and the Gromov-Witten theory of the Riemann sphere. Lett. Math. Phys. 53, 59-74 (2000)

14. Stanley, R.P.: Enumerative Combinatorics, vol. 2. Cambridge University Press, Cambridge (1999)

15. Witten, E.: Two dimensional gravity and intersection theory on moduli space. Surv. Differ. Geom. 1, 243-310 (1991)

16. Zelevinsky, A.V.: Representations of Finite Classical Groups, A Hopf Algebra Approach. Springer, Berlin (1981) 